\title{
Die Wiederherstellung des Rosengartens AM KÖNIGSUFER IN DRESDEN
}

\author{
Stephanie Jäger
}

$\mathrm{K}$

ann man mit Sondergärten Bauspekulationen auf Dauer verhindern? Stadtgartendirektors Heinri Balke (1903-1997) zu Beginn der 1930er Jahre in Dresden. Wir wissen heute, dass es möglich war.

Aber wie war die Situation zu dieser Zeit in der Region? Dresden war in aller Welt bekannt als Stadt der Kunst, der Musik und baulich als Stadt des Barock. Dies war der Eindruck der linkselbisch gelegenen Altstadt. Die Neustädter Seite Dresdens hingegen, die sich am gegenüberliegenden Elbufer befindet, bedurfte zu Beginn des 20. Jahrhunderts nach wie vor einer Aufwertung beziehungsweise einer städtebaulichen Neuordnung. Funktionalität prägte diesen Bereich. Hier reihten sich damals immer noch Ausschiffungsplätze aneinander. Das erforderte Lagerplätze für Holz, Kohle und Baumaterial. Auf dem Gelände des späteren Rosengartens, direkt am Flusslauf gelegen, befanden sich des Weiteren eine Wäscherei - damals wurde ja in der Elbe noch gewaschen - und die Sportplätze einer Schule. Heinrich Balke schlug dem Rat der Stadt vor, an deren Stelle Gärten zu favorisieren. Sein Ziel war es, mit aufwendig gestalteten Sondergärten entlang einer Uferpromenade jahrzehntelange Bestrebungen zur Bebauung zu verhindern. Er wollte gleichzeitig ein würdiges Pendant zur repräsentativen Altstädter Seite schaffen. So entstanden der Rosen- und der Staudengarten als Teile des sogenannten Königsufers. Dieser etwa zwei Kilometer lange Elbuferabschnitt zog sich von der Marienbrücke im Westen bis zur Prießnitzmündung im Osten und wurde $a b$
1933 unter der Leitung Balkes als großzügig gestaltete Promenade mit thematischen Sondergärten angelegt. Die Planung der Baulichkeiten übernahm der Dresdner Stadtbaurat Paul Wolf (1879-1957).

\section{Gliederung und Entstehung des Rosen- gartens}

Balke legte den Garten in folgender Weise an: Er teilte den langgestreckten Bereich in drei Teile: in einen Vorderteil, einen Mittelteil und einen Senkgarten mit Café. Man betrat den Garten zunächst von der Stadtseite, von Westen her. Der nördliche Brückenkopf der Albertbrücke bildete den Auftakt. Über eine Treppe gelangte man nach einer kurzen Wegstrecke in den ersten Gartenbereich. Hieran schloss sich das mit Rot-Eichen bestandene Trennungs- oder Zwischenstück mit dem Gärtnerdepot an. Es waren übrigens vermutlich die Umkleideräume der ehemaligen Sportplätze, die man belassen hatte. Dieser Bereich ist deutlich Richtung Elbe, also in der Querachse ausgerichtet. Entlang der maßgebenden Hauptachse gelangte man in den Mittelteil mit Hochstamm- und Beetrosen. An diesen grenzte der schmale Rittersporngarten an. Über eine weitere Treppe kam man nun in den Senkgarten mit dem Café-Gebäude als räumlichen Abschluss des Gartens in der Längsachse. Den Grundstock des Cafés bildete die Wartehalle der "Sächsisch-Böhmischen Dampfschiffahrts-Gesellschaft«.

Elbseitig wurde die Anlage durch eine Allee aus Rotdorn, den sogenannten Crataegusweg, begrenzt. Die einzelnen Quar- 
tiere waren jeweils mit Hainbuchen-Hecken (Carpinus betulus) eingefasst.

Der Baubeginn des Rosengartens lag im Jahr 1933. Zwei Jahre später konnte er in Teilbereichen eröffnet werden. Weitere Arbeiten wurden bis 1936 vorgenommen. $\mathrm{Zu}$ diesem Zeitpunkt fand in Dresden die Reichsgartenschau statt. Heinrich Balke plante hier verschiedene Bereiche selbst, so auch den dortigen Rosengarten und hatte für das gesamte Projekt die gartenkünstlerische und fachliche Leitung. Mit Beendigung der Schau konnte er veranlassen, dass Materialien, Pflanzen und Ausstattungselemente wie Skulpturen in den Rosengarten übernommen und gestalterisch integriert wurden. Das war für ihn unter anderem auch ein entscheidender wirtschaftlicher Faktor und eine Erklärung dafür, dass wir hier nicht nur heimische Materialien vorfinden, sondern beispielsweise auch Wesersandstein oder Theumaer Schiefer.

Der Garten hatte nach der Fertigstellung eine Gesamtgröße von 500 m x 60 m, mit einem Bestand von 6.000 Rosenstöcken. Er diente damals als Lehr- und Schaugarten. Heute steht die Anlage unter Denkmalschutz und befindet sich in Verwaltung des Amtes für Stadtgrün und Abfallwirtschaft Dresden.

\section{Zustand vor Beginn der Restaurierung ab 1995}

Die entscheidende Zäsur für die Anlage war das Jahr 1945. Mit der Zerstörung Dresdens wurde auch der Rosengarten stark beschädigt. Instandsetzungsarbeiten größeren Umfangs erfolgten in den Jahren 1950 und 1970 jeweils im Geschmack der Zeit. In Teilbereichen hatte das den Verlust der ursprünglichen Gestaltung sowie räumlicher Strukturen zur Folge. Zu Beginn der 1990er Jahre musste auch ein großer Teil der Rosen als überaltert eingestuft werden.

Bodenmüdigkeit führte zu Ausfällen des Bestandes. Durch willkürliche Nachpflanzungen über einen langen Zeitraum hinweg fehlte eine erkennbare farbliche Konzeption. Plattenwege und Ausstattungselemente waren desolat, die Kunstwerke entweder beschädigt, während des Krieges entfernt und eingeschmolzen oder umgesetzt worden. Ein weiteres Problem waren willkürliche Gehölzpflanzungen und Wildwuchs in den Randbereichen. Dadurch gingen wichtige und beabsichtigte Blickbeziehungen in den angrenzenden Landschaftsraum verloren und gaben dem Garten eine von Heinrich Balke so nicht beabsichtigte räumliche Enge.

\section{Denkmalpflegerische Zielstellung}

Um die Pflege zu optimieren, wurde 1995 der Hauptstrang für ein modernes Bewässerungssystem in der gesamten Längsachse des Gartens verlegt. Der geplante weitere differenzierte Ausbau beinhaltete, dass generell alle Rosen aus den Beeten genommen werden sollten. Gleichzeitig wurde es als Chance gesehen, nach über 60 Jahren einen Bodenaustausch vorzunehmen. Diese technischen Aspekte lösten die Frage nach der historischen Situation sowie Recherchen beziehungsweise denkmalpflegerische Analysen aus.

In der städtischen Bildstelle konnten eine Fotoplatte mit dem Entwurfsplan von Heinrich Balke (Abb. 1) sowie einige historische Aufnahmen gefunden werden. Wesentliche Veränderungen in der Grundstruktur des Gartens waren hier bereits erkennbar. Für eine denkmalgerechte Wiederherstellung waren diese Unterlagen allerdings nicht ausreichend. Man kann davon ausgehen, dass alle Detailpläne mit der Zerstörung des Gärtnerdepots 1945 verbrannten.

1997 erfolgte deshalb durch unser Amt eine intensive Öffentlichkeitsarbeit, verbunden mit dem Aufruf an die Dresdner Bevölkerung, durch private historische Aufnahmen die Arbeit des damaligen Grünflächenamtes zu unterstützen. Fast 60 Bürger haben daraufhin ihre Unterlagen wie Fotos und Postkarten zur Verfügung gestellt. Es ergaben sich hilfreiche Kontakte zum Sohn des 


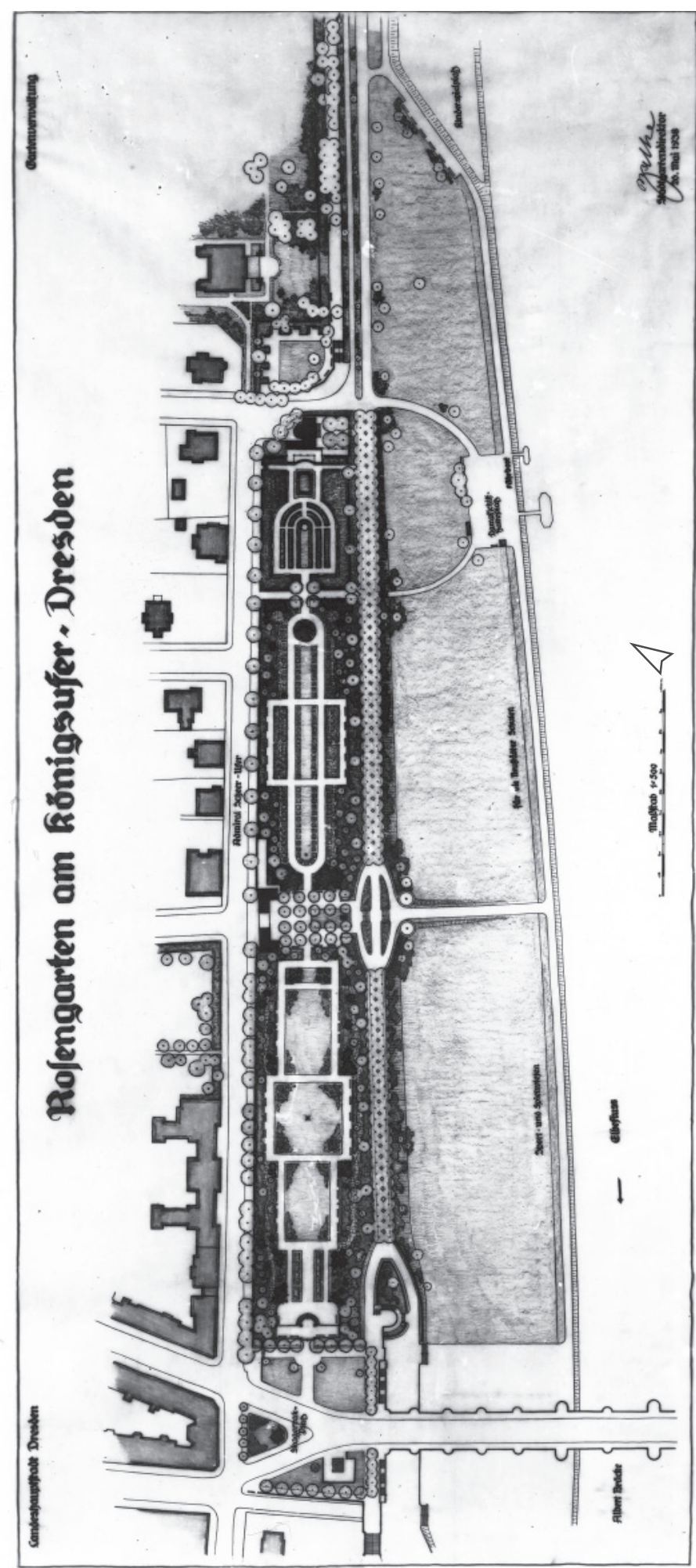

1 | Entwurfsplan, Heinrich Balke 1938 (Landeshauptstadt Dresden, Bildstelle). 


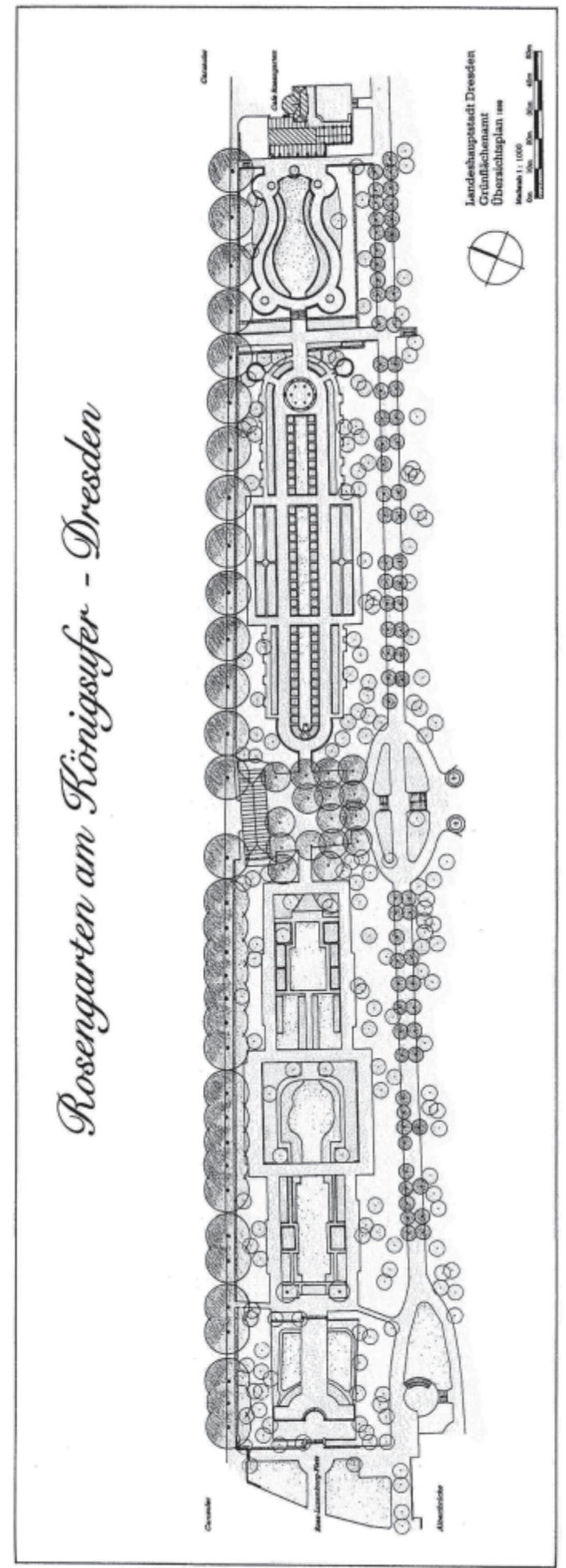

2 | Übersichtsplan vor Rekonstruktion, Susann Richter 1999 (Amt für Stadtgrün und Abfallwirtschaft Dresden). 
ehemaligen Obergartenmeisters Hans Graumann und zur Witwe von Heinrich Balke. Somit hatten wir einen guten und umfangreichen visuellen Fundus für die geplante Restaurierung des Gartens. Die Auswertung der Bilder verdeutlichte detaillierter die wesentlichen Veränderungen. In Abstimmungen mit dem Landesamt für Denkmalpflege Sachsen wurde entschieden, in den zunächst geplanten Restaurierungsabschnitten im Vorder- und Mittelteil so weit wie möglich auf die ursprüngliche Gestaltung der 1930er Jahre zurück zu gehen.

\section{Wiederherstellung des Gartens}

Es folgt jetzt eine Gegenüberstellung der einzelnen Gartenbereiche in der Ausgangssituation, das heißt in den 1930er Jahren sowie vor (Abb. 2) und nach der Restaurierung ab 1995.

\section{Vorderteil}

Die stadtseitige Eingangssituation in den
Vorderteil des Gartens war einst zeitgemäß durch eine repräsentative Treppenanlage geprägt (Abb. 3). Heute sind hier nur noch drei Stufen vorhanden. Die Erklärung ist, dass nach 1945 großflächig Trümmerschutt der angrenzenden Wohnbebauung mit einer Höhe von ca. $50 \mathrm{~cm}$ aufgetragen wurde. Das führte zur ersten entscheidenden Veränderung der Anlage. Eine Rückführung auf das ursprüngliche Geländeniveau wird bis heute aus Kostengründen nicht in Erwägung gezogen. Dieses kleinere Areal ist bisher als einziger Bereich des Rosengartens noch unsaniert.

Der anschließende Hauptraum des Vorderteils wurde historisch mit wenigen Rosensorten gestaltet und weiträumig angelegt. Hier befanden sich drei Rasenspiegel, in deren Eckpunkten vorrangig flächige Strauchrosenpflanzungen angeordnet waren. In den 1950er Jahren erfolgte eine grundsätzliche räumliche Umgestaltung. Es wurden Plattenwege sowie Beetrosen, Stauden und Gehölze eingebracht. Die Einschränkung der Raumwirkung sowohl innerhalb des Gar-

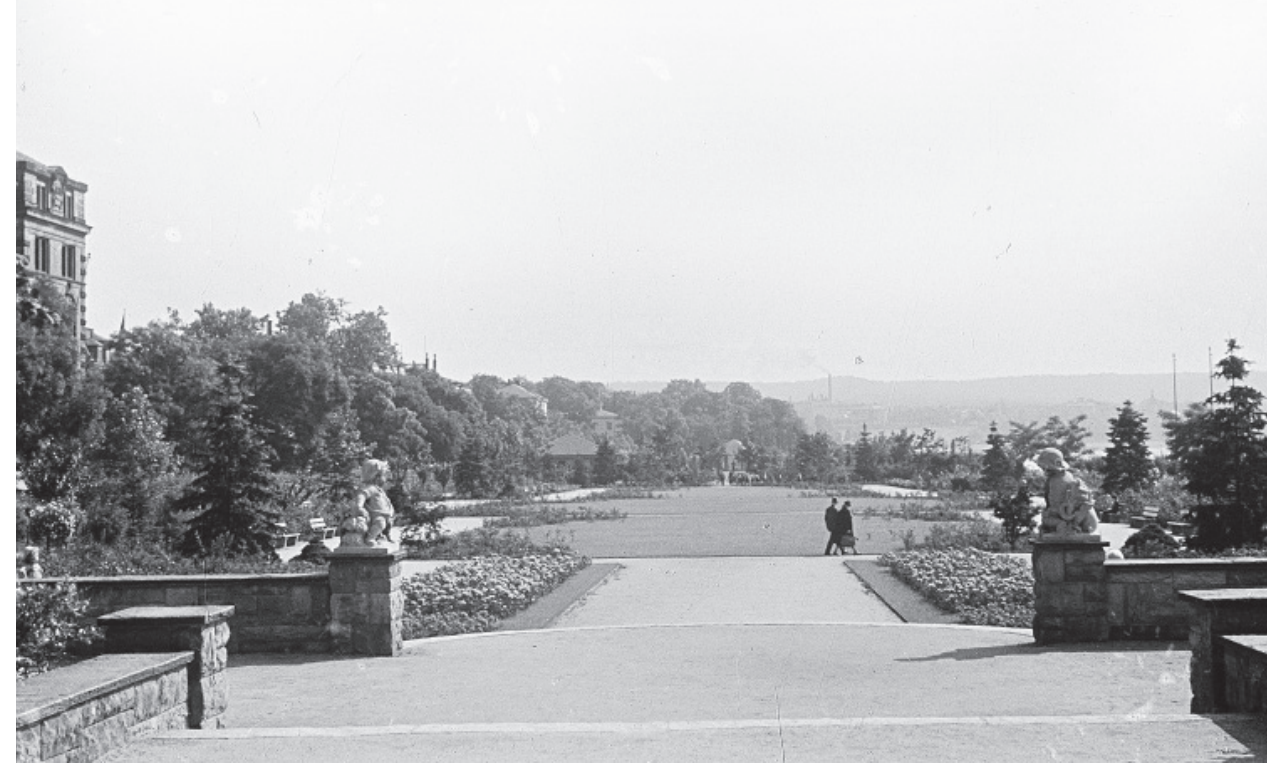

3 | Vorderteil, Fotografie, Hans Graumann um 1938 (Amt für Stadtgrün und Abfallwirtschaft Dresden, Archiv). 


\section{Die Wiederherstellung des Rosengartens am Königsufer in Dresden}

tens als auch in den Landschaftsraum war die Folge.

Vor Beginn der Wiederherstellung erfolgte auf der Grundlage einer Zustandsüberlagerung des »Balke-Planes« mit der gegebenen Situation eine Absteckung vor Ort. So konnte die Überlegung zur Rückführung auf die 1930er Jahre, insbesondere der immense Umfang des Vorhabens, besser beurteilt und letztendlich bestätigt werden.

Begonnen wurde mit der Bergung verwendbarer Materialien, Stauden und Gehölze. Die Eckpunkte der drei Rasenspiegel wurden wieder flächig mit Rosenpflanzungen sowie Solitärgehölzen wie Fächer-Ahorn (Acer palmatum) versehen. Hierbei kamen analog historischer Fotos Strauchrosen in der Farbkombination Gelb in der Mitte und Rosa in der Umpflanzung zur Anwendung. Die Sortenauswahl lehnte sich an die Zeit zwischen 1900 und 1937 an. Fünf KoloradoTannen (Abies concolor) konnten in Auswertung des Fotomaterials am originalen Standort nachgepflanzt werden und tragen nun wieder entscheidend zur Raumbildung bei
(Abb. 4). Das restauratorische Ziel, sich optisch der Situation der 1930er Jahre weitestgehend anzunähern, konnte erreicht werden.

\section{Mittelteil}

Im Mittelteil des Rosengartens (Abb. 5) sind die Grundstrukturen erfreulicherweise erhalten geblieben. Verloren gegangen war aber die historische ausgewogene Farbkomposition. Störend waren auch die Waschbetonborde, welche man zur Erleichterung der Pflege in den 1970er Jahren eingebaut hatte. Diese wurden heraus genommen und die ursprüngliche Situation wieder hergestellt. Analog zum Vorderteil erfolgten ein Bodenaustausch bis zu einer Tiefe von $60 \mathrm{~cm}$ und gleichzeitig die Installation einer Beregnungsanlage. Nach einer Zwischenbegrünung mit Tagetes wurde ein Sortiment von sächsischen Beetrosen der 1970er und 1980er Jahre angepflanzt, dem die Farbkomposition der 1930er Jahre zu Grunde liegt. Da diese Rosen kaum noch verfügbar sind, wurde ein spezieller und langjähriger

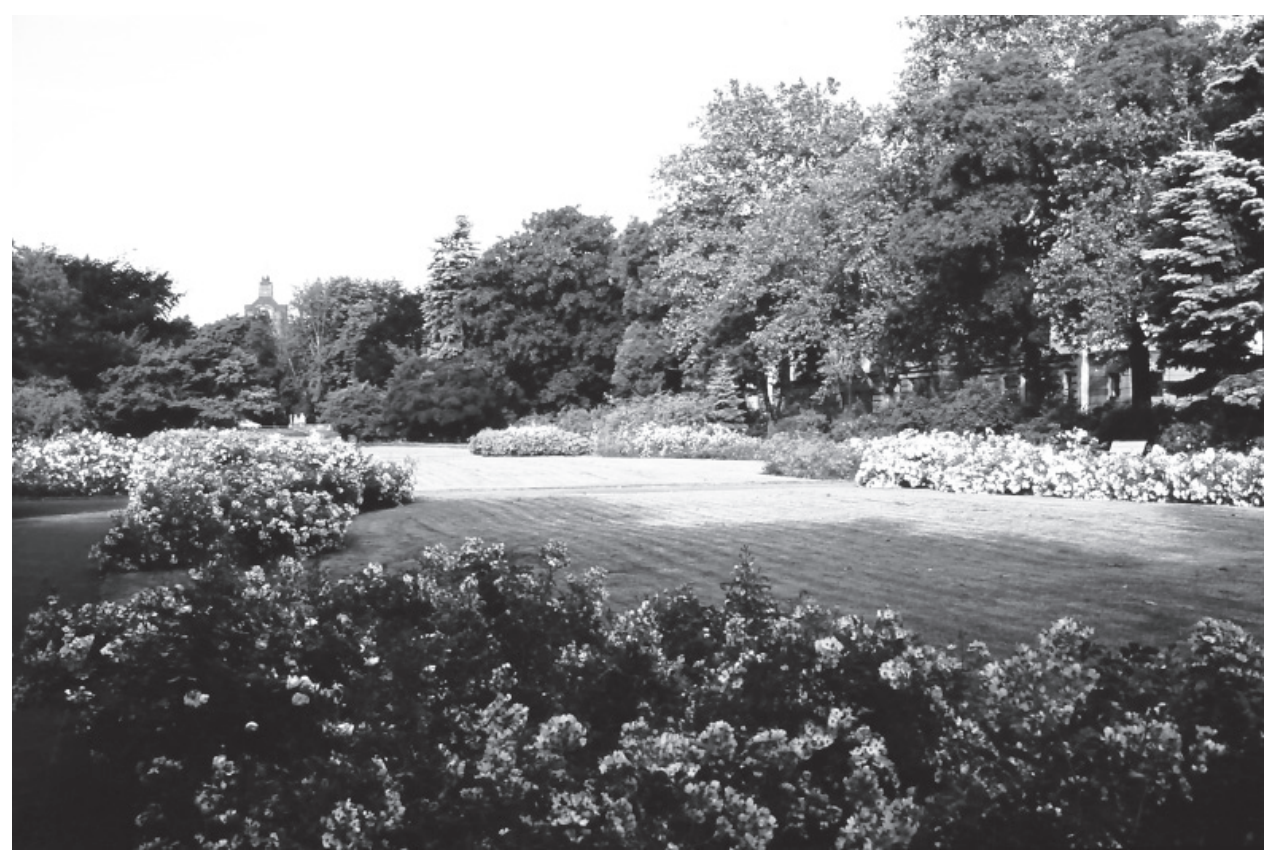

4 | Vorderteil, Fotografie, Stephanie Jäger 2016 (privat). 
Anzuchtsvertrag mit einer sächsischen Baumschule abgeschlossen. Der Erhalt dieser DDR-Sorten ist für uns ein wichtiger Aspekt praktizierter Gartendenkmalpflege.

\section{Rittersporngarten}

2001/2002 konnte die Wiederherstellung des Rittersporngartens realisiert werden. Es ist ein relativ kleiner Bereich, der aber aufgrund seiner Farbkomponente einen besonders wichtigen Aspekt im Gesamtkontext der Anlage darstellt. Er war in seiner ursprünglich vorgegebenen Thematik nicht mehr vorhanden. Statt des Rittersporns hatte man Rosenbeete mit Liegebordeinfassung angelegt, eine Hecke gerodet, damit eine Öffnung der Sicht in Richtung Senkgarten geschaffen und die überdimensionierten Bankplätze entsprechend ausgerichtet.

Zunächst konnte die in den 1970er Jahren gerodete Hainbuchen-Hecke nachgepflanzt werden. Damit wurde wieder Balkes grundsätzlichem Gestaltungsprinzip entsprochen. Auf Grund der zu diesem Zeitpunkt fehlen- den Finanzierung wurden Carpinus-Sämlinge aus dem Großen Garten geborgen und mit den vor Ort tätigen Gärtnern aufgepflanzt. Die Hecke ist hervorragend angewachsen und inzwischen auf der historischen Höhe in Form gebracht. Die einzelnen Rittersporn-Sorten wurden aus dem Sortiment von Karl Foerster aus der Zeit der 1930er Jahre ausgewählt. Die Standorte der Bankplätze wurden entsprechend korrigiert.

\section{Senkgarten}

2005/2006 begannen die Sanierungsarbeiten im Senkgarten, dem ältesten Teil des Rosengartens (Abb. 6). 1976 war hier anlässlich der 16. Arbeiterfestspiele der DDR eine Umgestaltung in einer dem Garten nicht adäquaten Formensprache erfolgt. Die fachlich favorisierte Rückführung auf die ursprüngliche Situation konnte leider nicht durchgeführt werden, denn seit dem Verkauf des Cafés am Rosengarten einschließlich umgebender Flächen an einen privaten Eigentümer befinden sich für eine Rekon-

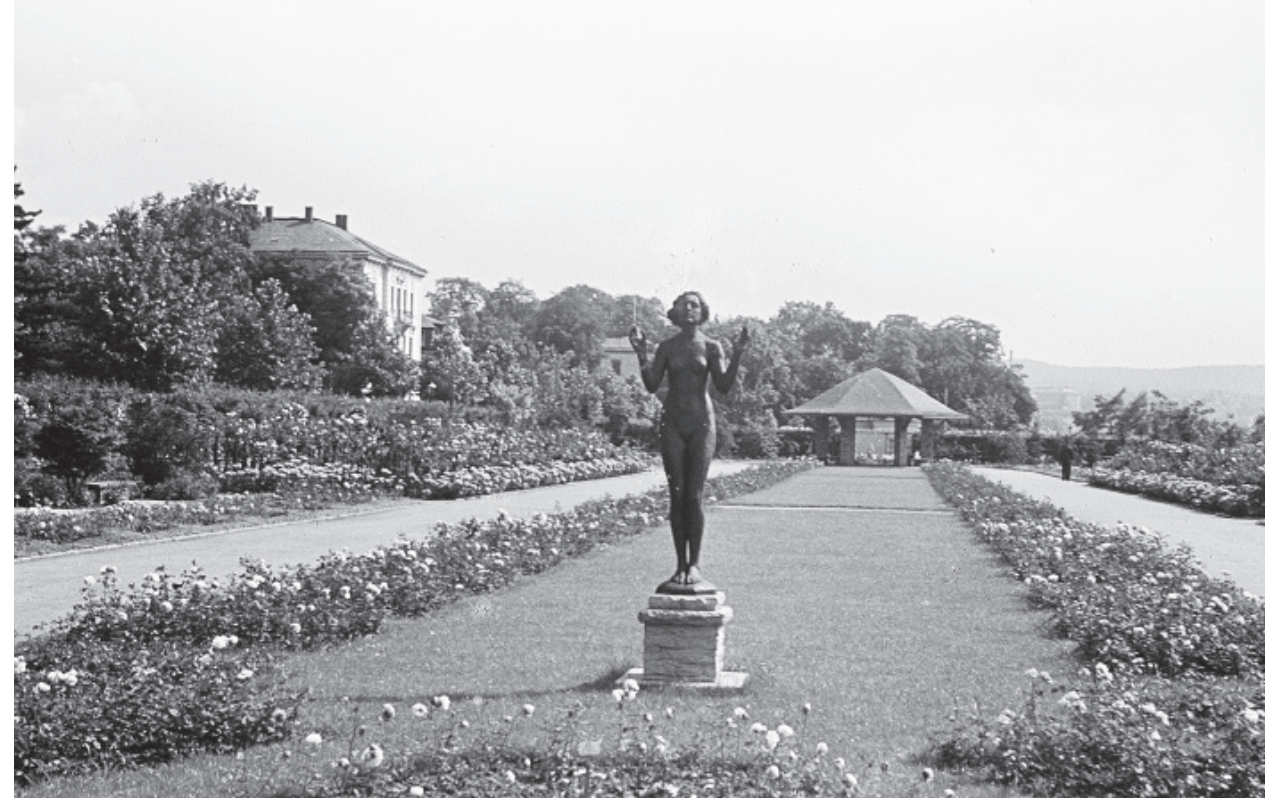

5 | Mittelteil, Fotografie, Hans Graumann um 1938 (Amt für Stadtgrün und Abfallwirtschaft Dresden, Archiv). 


\section{Die Wiederherstellung des Rosengartens am Königsufer in Dresden}

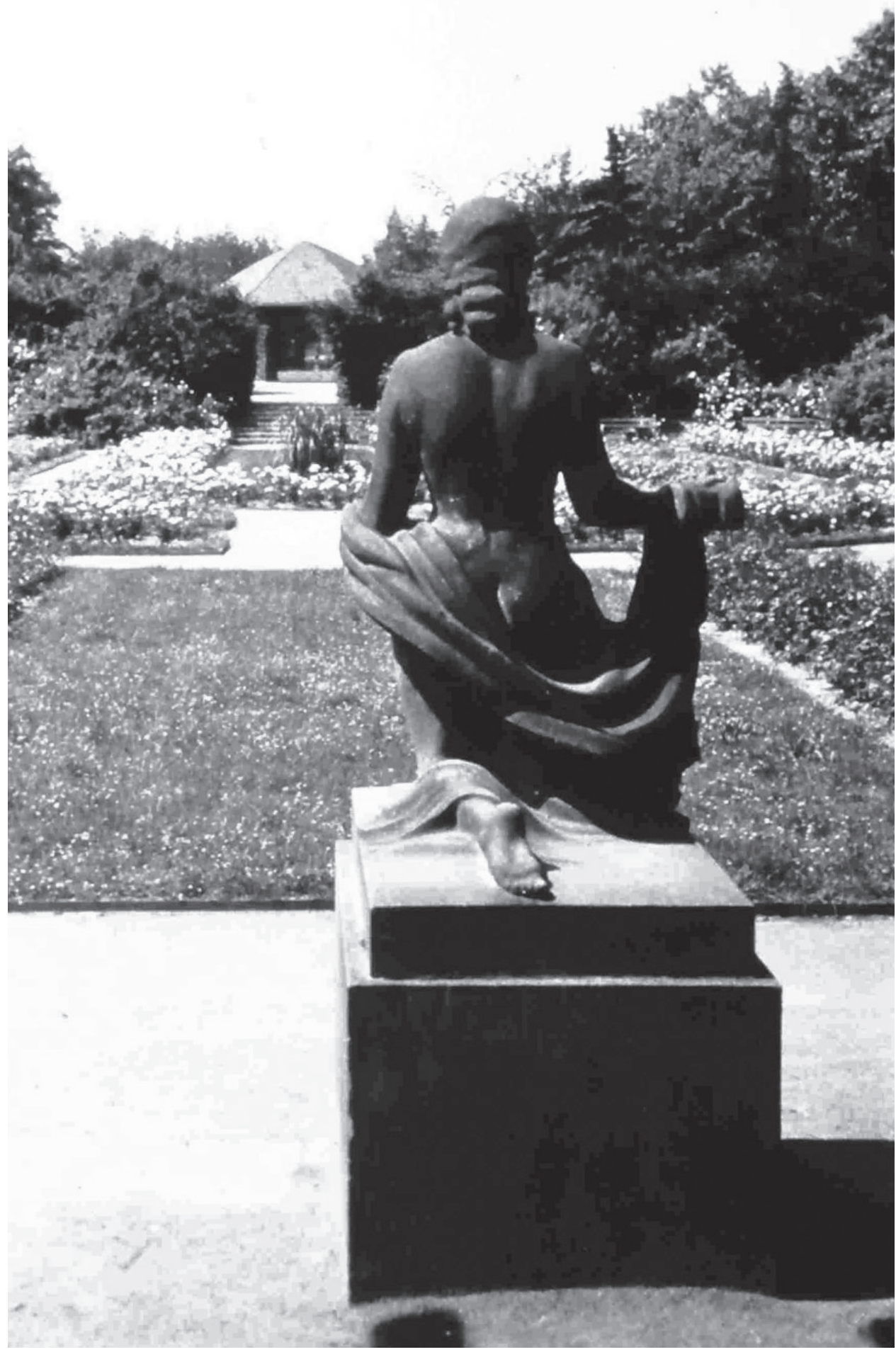

6 | Senkgarten mit der Plastik »Kniende« von Otto Rost, Fotografie, Hans Graumann um 1938 (Amt für Stadtgrün und Abfallwirtschaft Dresden, Archiv). 
struktion unabdingbare Teilbereiche nicht mehr im kommunalen Eigentum. So beschränkte sich das Fachamt auf die Herausnahme desolater Asphaltwege und deren Ersatz durch sandgeschlämmte Decken, Reparaturarbeiten sowie Nachpflanzungen von Gehölzen und Rosen.

\section{Crataegusweg}

In den darauffolgenden zwei Jahren begann die Wiederherstellung der elbseitigen Allee, des Crataegusweges. Hier waren ein Restbestand der historischen Altbaumsubstanz an den jeweiligen Stirnseiten, Fehlstellen und Nachpflanzungen mit Japanischer BlütenKirsche (Prunus serrulata 'Kanzan') zu verzeichnen. In den 1980er Jahren erhielt die Stadt Dresden Zier-Kirschen als Geschenk aus Japan, welche auch hier zur Anpflanzung kamen. Im Zuge der Restaurierung wurde wieder Rotdorn (Crataegus laevigatus 'Paul's Scarlet') gepflanzt. Vier Bestandsbäume blieben als Dokumentationsbäume erhalten. Der Crataegusweg präsentiert sich jetzt mit einem einheitlichen Erscheinungsbild. Er bildet als Promenadenweg den elbseitigen Abschluss des Rosengartens. Balke bezeichnete diesen gleichzeitig als Bindeglied beziehungsweise Gelenk zwischen der Stadt und der freien Landschaft.

Nicht unerwähnt bleiben soll die Problematik der elbnahen Lage. Der Senkgarten hat die ursprüngliche Geländehöhe, alle anderen Gartenbereiche konzipierte Balke entsprechend der damaligen Parameter vorausschauend hochwassersicher und ließ das Gelände aufschütten. Leider entspricht das bekanntermaßen nicht mehr den jetzigen Gegebenheiten. 2002 war der Rosengarten sehr stark vom Hochwasser betroffen. Wege, bauliche und vegetabile Ausstattung wurden so gut wie möglich gereinigt, entschlammt und instand gesetzt. Auch nach dem Hochwasser 2013 waren Schäden zu verzeichnen, wenn auch in geringerem Ausmaß. Hier sind die Wiederherstellungsarbeiten noch nicht abgeschlossen.

\section{Künstlerische Ausstattung}

Die Themen Rose und Kunst stehen in allen Gartenbereichen im engen Zusammenhang. Wir finden vor allem Darstellungen von Tieren und weiblichen Figuren. Die Materialauswahl ist sehr unterschiedlich und reicht von Muschelkalk über Sandstein bis hin zu Bronze. Ein besonderes thematisches Programm lag wahrscheinlich nicht vor. Heinrich Balke war daran gelegen, so übermittelte es uns seine Witwe, Künstler zu fördern und ihnen in wirtschaftlich schwierigen Zeiten die Möglichkeit des Broterwerbs zu geben. Nach 1990 wurden die Kunstwerke in die Wiederherstellung des Gartens einbezogen. Je nach Situation ergaben sich verschiedene restauratorische Ansätze.

Erstes Beispiel sind zwei Bärenplastiken des Bildhauers Rudolf Löhner (1890-1971), welche nach 1945 im Dresdner Zoo zur Aufstellung kamen. In Auswertung der historischen Fotos konnten diese den Sitzrondellen im Rosengarten zugeordnet werden. Im Jahr 2000 bestand die Möglichkeit, sie an den Originalstandort zurückzuholen. Sie befinden sich jetzt wieder am angestammten Platz in den sanierten Rondellen des Mittelteils.

Ein weiteres Kunstwerk, der »Stier « von Ernst Moritz Geyger (1861-1941), befand sich ursprünglich im Zwischenstück nahe dem Gärtnerdepot mit Ausrichtung zur Elbe. Das Kunstwerk gilt als Kriegsverlust. Für den seit 1945 leer stehenden Sockel wurde in Zusammenarbeit von Landesamt für Denkmalpflege Sachsen und der Kunstkommission der Stadt Dresden ein künstlerischer Wettbewerb ausgelobt. Einzige Vorgabe war der Erhalt des Sockels in seiner überlieferten Größe. Dieser wurde saniert und im Ergebnis die moderne Plastik "ani-mal« des Bildhauers Steffen Bachmann (geb. 1962) aufgestellt. Den Umgang mit den weiteren Kunstwerken möchte ich nun kurz erläutern.

Die vier Putten aus Muschelkalk des Bildhauers Max Hermann Fritz (1873-1948) 
wurden gereinigt und Fehlstellen ergänzt.

Die Figur "Genesung " von Felix Georg Pfeifer (1871-1945), deren Erstguss sich in Leipzig vor dem Hauptgebäude der AOK befunden hatte, war nach 1945 nicht mehr existent. Ursprünglich war sie ein Exponat der Reichsgartenschau auf dem Gelände des Großen Gartens gewesen und wurde »Beglückende Schönheit« genannt. 1937 erfolgte die Umsetzung in den Rosengarten, nunmehr als "Genesung «. Die AOK hatte in den 1990er Jahren Interesse gezeigt, die Brunnenfigur in Leipzig wieder herzustellen. Die Dresdner Plastik wurde für einen Abguss ausgeliehen und im Gegenzug wurde diese für uns kostenfrei gereinigt und restauriert.

Das "Mädchen mit Gazelle« von Georg Wrba (1872-1939) gilt als Kriegsverlust. Mit der Umgestaltung des Senkgartens wurde die hier einst in der Hauptachse stehende "Kniende" von Otto Rost (1887-1970) auf den freien Sockel umgesetzt. Diese Figur wurde ebenfalls gereinigt und Fehlstellen ergänzt. Am ursprünglichen Standort befindet sich seit den 1970er Jahren eine Wasserschale.

\section{Pflege}

Die Pflege erfolgt im Auftrag unseres Amtes durch den Regiebetrieb Zentrale Technische Dienstleistungen Dresden. Drei Mitarbeiter sind vor Ort tätig. Das Gärtnerdepot befindet sich im Zwischenstück des Rosengartens. Es ist eine Fläche von $30.286 \mathrm{~m}^{2} \mathrm{zu}$ unterhalten. Die Kosten belaufen sich auf $163.500 €$ pro Jahr.

\section{Fazit}

Die Wiederherstellungsarbeiten sind weitestgehend abgeschlossen. Zusammenfassend kann man sagen, dass der gartenrestauratorische Ansatz je nach Ausgangssituation in den einzelnen Teilbereichen der Anlage sehr differenziert war: Der konsequente Rückbau der 1950er Jahre im Vorderteil, das Untersetzen des auf Farben angelegten Mittelteils mit einem DDR-Rosensortiment und der Beibehalt der 1970er Jahre im Senkgarten aufgrund des Flächenverkaufs.

Stadtgartendirektor Heinrich Balke konnte seine Visionen einer gestalteten Uferpromenade verwirklichen. Er war übrigens in den 1950er und 1960er Jahren in Wuppertal wieder als Amtsleiter tätig, betrachtete aber die Gestaltung des Rosen- und Staudengartens als sein eigentliches Lebenswerk. Unter dem Blickwinkel der sowohl politisch, als auch wirtschaftlich schwierigen 1930er Jahre wird das Königsufer heute als die bedeutendste städtebauliche und gärtnerische Leistung dieser Zeit in Dresden eingestuft. 